\title{
A novel curcumin-loaded composite dressing facilitates wound healing due to its natural antioxidant effect
}

This article was published in the following Dove Press journal:

Drug Design, Development and Therapy

\author{
Yong Zhao' \\ Chuanyu Dai ${ }^{2}$ \\ Zheng Wang' \\ Weimin Chen' \\ Jiaguo Liu $^{3}$ \\ Renxi Zhuo ${ }^{2}$ \\ Aixi Yu' \\ Shiwen Huang ${ }^{2}$ \\ 'Department of Orthopedic Trauma and \\ Microsurgy, Zhongnan Hospital of Wuhan \\ University, Wuhan 430070, Hubei, \\ People's Republic of China; ${ }^{2}$ Key \\ Laboratory of Biomedical Polymers of \\ Ministry of Education, Department of \\ Chemistry, Wuhan University, Wuhan, \\ Hubei 430072, People's Republic of \\ China; ${ }^{3}$ Department of Orthopedics, \\ Hubei University of Medicine Affliated \\ Taihe Hospital, Shiyan 442000, Hubei, \\ People's Republic of China
}

Correspondence: Aixi Yu

Department of Orthopedic Trauma and Microsurgy, Zhongnan Hospital of Wuhan University, 169 Donghu Road, Wuchang District, Wuhan 430070, Hubei, People's Republic of China

$\mathrm{Tel}+86$ I 3507187489

Email yuaixi@whu.edu.cn

Shiwen Huang

Key Laboratory of Biomedical Polymers of Ministry of Education, Department of Chemistry, Wuhan University, 16

Luojiashan Road, Wuchang District,

Wuhan 430072, Hubei, People's Republic of China

Tel +86 I86 27865822

Email swhuang@whu.edu.cn
Purpose: To prepare a novel wound dressing to facilitate cutaneous wound healing.

Methods: Curcumin (Cur) was added to the ring-shaped $\beta$-cyclodextrin (CD) to form a $\beta$-CD-Cur inclusion complex (CD-Cur). CD-Cur was then integrated into a composite chitosan-alginate (CA) mix. Finally, CA-CD-Cur was generated with a freeze-drying technique. Water-uptake capacity, degradation rate, and drug-release kinetics of the newly formed dressing were investigated in vitro. In animal studies, cutaneous wounds in rats were created, treated with CA-CD-Cur, then compared to CA-Cur, CA, and gauze.

Results: CA-CD-Cur-treated wounds showed accelerated closure rates, improved histopathological results, and lower SOD, lipid peroxidation, pI3K, and pAktkt levels than other groups. On the contrary, catalase, $\mathrm{I} \kappa \mathrm{B} \alpha$, and $\mathrm{TGF} \beta_{1}$ levels were higher than others.

Conclusion: CA-CD-Cur may facilitate cutaneous wound dressing that facilitate wound healing.

Keywords: curcumin, cyclodextrin inclusion complex, chitosan, alginate, cutaneous wound healing

\section{Introduction}

Wound healing can be divided into three distinct but interrelated phases: inflammation, proliferation, and remodeling. Orchestrated reactions and interactions between cells and chemicals are involved in the healing process. During the inflammation phase, especially in patients with an imbalanced internal environment, excessive inflammatory mediators such as radicals are released to the wound site. ${ }^{1,2}$ These radicals are often associated with oxidative stress and subsequent prolonged inflammation, resulting in impaired wound healing. ${ }^{1,3}$

Curcumin (Cur), the active component of turmeric, has long been used in India, China, and other Southeast Asian countries to treat a variety of diseases, due to its antioxidant, anti-inflammatory, and anticarcinogenic properties. ${ }^{1,3-5}$ Studies have shown that Cur may assist cutaneous wound healing, even in troublesome conditions, such as irradiated wounds, burns, laser-induced wounds, and diabetic wounds. ${ }^{6,7}$ Despite its remarkable biological effects, Cur's extremely low water solubility severely limits its bioavailability and clinical efficacy.

The cyclodextrin (CD)-Curcomplex is a modification to enhance Cur's water solubility. In CD-Cur, Cur is included inside cyclic CD molecules, which are frequently used as solubilizing and stabilizing agents in pharmaceutical preparations. $^{8,9} \mathrm{CD}$-Cur has been used in cancer therapy, and exhibited superior 
effects to Cur alone in animal experiments. ${ }^{10}$ Possible mechanisms involved may be higher cellular uptake and longer half-life in the cells. ${ }^{11}$ Also, transdermal application of Cur-CD showed higher skin permeability compared with free Cur in a specific skin model. ${ }^{12}$ These results suggest that with proper preparation, Cur-CD might be a suitable component for cutaneous wound healing.

Chitosan and alginate are both promising materials as wound-dressing materials, due to their bioactive, hemostatic, and bacteriostatic properties. These dressings can maintain a moist environment and stimulate granulationtissue formation when applied topically on cutaneous wounds. Through chemical binding and freeze-drying, chitosan and alginate can form polyelectrolytes with porous, net-like structure (chitosan-alginate [CA]), which functions as a qualified drug-loading material for cutaneous wound dressing. ${ }^{1,13-15}$

Researchers have loaded Cur into CA to form a composite material, CA-Cur, which showed promising woundhealing capacity. ${ }^{13}$ In this study, instead of Cur, CD-Cur was loaded into $\mathrm{CA}$ to form a relatively new wounddressing material (CA-CD-Cur). We hypothesized that the improved water solubility and bioactivity of CD-Cur would make CA-CD-Cur a more suitable material in treating cutaneous wounds. The physical and chemical profile of the composite sponge was characterized. After that, sponges were subjected to in vivo animal experiments to evaluate their therapeutic efficacy. Wound appearance, wound-closure rate, and histopathological examinations were used to assess the healing effect of CA-CD-Cur. Possible mechanisms involved in this process were also investigated at both protein and gene levels.

\section{Methods}

$\beta$-CD, Cur (Sigma-Aldrich), chitosan (degree of deacetylation $85 \%$, high viscosity [ $>400 \mathrm{mPas}$ ], $179.17 \mathrm{MW}$ ), sodium alginate (AR assay $\geq 98 \%$, viscosity $\left[10 \mathrm{~g} / \mathrm{L}, 20^{\circ} \mathrm{C}\right] \geq 0.02$ $\mathrm{mm}^{2} /$ second), acetoneused in this experiment were analytical reagent grade. Female Sprague Dawley rats weighing 200$220 \mathrm{~g}$ were purchased from the Laboratory Animal Center of Wuhan University, China. Animals were housed singly in a laminar flow operating room with a 12-hour light-12-hour dark cycle and fed sterile food and water ad libitum. Treatment of the animals was carried out in strict accordance with the recommendations described in the Guide for the Care and Use of Laboratory Animals of the National Institutes of Health. The experimental protocol was approved by the Committee on the Ethics of Animal Experiments of Wuhan University, Wuhan, People's Republic of China.

\section{Preparation of $\beta$-cyclodextrin-curcumin complexes}

CD-Cur was prepared with a self-assembly method by mixing $C D$ and Cur at a molar ratio of $1: 1{ }^{10}$ Briefly, $\beta-C D(647 \mathrm{mg}$ ) was dissolved in $120 \mathrm{~mL}$ deionized water in a $200 \mathrm{~mL}$ glass vial with a magnetic bar, and $147 \mathrm{mg}$ Cur containing $2 \mathrm{~mL}$ acetone was then added to the CD solution while stirring at $400 \mathrm{rpm}$. The solution was stirred for 24 hours in a fume cupboard with no cap to evaporate the acetone hidden from light, and centrifuged at $1,000 \mathrm{rpm}$ for 5 minutes. After that, the supernatant was collected and freeze-dried (Labconco Freeze Dry System) to recover water-soluble CD-Cur. Physicochemical characterization of CD-Cur can be found in Figure S1.

\section{Preparation of chitosan-alginate sponge}

For preparation of $\mathrm{CA}$, alginate and chitosan were mixed at a $1: 1$ ratio (w:v) based on a former study. ${ }^{13}$ According to that research, compare to other tested ratios $(1: 3,3: 1)$, the $1: 1$ ratio for the $\mathrm{CA}$ composite exhibited the best overall performance. Alginate solution (1\% w:v) was prepared by dissolving sodium alginate $(0.2 \mathrm{~g})$ in $20 \mathrm{~mL}$ deionized water. Meanwhile, chitosan solution (1\% CA sponge: preparation and application in Cur delivery for dermal wound healing in rat) was prepared by dissolving chitosan $(0.2 \mathrm{~g})$ in $20 \mathrm{~mL}$ deionized water with $0.2 \mathrm{~mL}$ acetic acid added. The CA solution was then mixed by gently adding chitosan solution to alginate solution with mechanical stirring (1,000 rpm, 10 minutes). The resultant mixture was centrifuged (to remove the bubbles), cast into a six-well plate, frozen overnight $\left(-80^{\circ} \mathrm{C}\right)$, and then lyophilized at $-35^{\circ} \mathrm{C}$ (VirTis Advantage Wizard 2.0) for 3 days.

\section{Preparation of CA-Cur and CA-CD-Cur sponges}

For preparation of CA-Cur and CA-CD-Cur, similar procedures were followed, except varying doses of Cur or CD-Cur were dissolved and added to alginate solution before mixing. In brief, for preparation of CA-Cur, 6.25 $\mathrm{mg}, 12.5 \mathrm{mg}, 25 \mathrm{mg}, 50 \mathrm{mg}$, and $75 \mathrm{mg}$ Cur were dissolved in $1.5 \mathrm{~mL}$ absolute alcohol and then added to chitosan solution separately. Similarly, for preparation of CA-CD-Cur, $37.47 \mathrm{mg}$ (containing $6.25 \mathrm{mg}$ Cur, based on 
the Cur content in CD-Cur; detailed calculation in Supplementary materials), $74.94 \mathrm{mg}$ (containing $12.5 \mathrm{mg}$ Cur), $149.88 \mathrm{mg}$ (containing $25 \mathrm{mg}$ Cur), $299.77 \mathrm{mg}$ (containing $50 \mathrm{mg}$ Cur), and $449.65 \mathrm{mg}$ (containing 75 $\mathrm{mg}$ Cur) of CD-Cur were dissolved into $1.5 \mathrm{~mL}$ deionized water and then added to chitosan solution separately.

\section{Water-uptake capacity}

The water-uptake capacity of CA, CA-Cur, and CA-CDCur was determined following the protocol of Dai et al. ${ }^{13}$ In brief, a known weight $\left(\mathrm{W}_{0}\right)$ of sponge was placed in PBS for 10 minutes to saturate the sponge with PBS. After that, filter paper was used to remove the excess water on the surface. The wet sponge was then weighed (Ww):

water-uptake capacity $(\%)=\left(\left[\mathrm{Ww}-\mathrm{W}_{0}\right] / \mathrm{Ww}\right) \times 100$

Six repetitions of of this procedure were done for each type of dressing.

\section{In vitro degradation}

Degradation profiles of CA, CA-Cur, and CA-CD-Cur were determined following the protocol of Dai et al. ${ }^{13}$ In brief, a known weight $\left(\mathrm{W}_{0}\right)$ of sponge was placed in PBS containing 500-1,000 U/ $\mathrm{cm}^{3}$ lysozyme. At specific time intervals, the sponges were taken out, washed, frozen, lyophilized, and then weighed:

$$
\text { weight loss }(\%)=\left(\left[\mathrm{W}_{0}-\mathrm{Wt}\right] / \mathrm{W}_{0}\right) \times 100
$$

Six repetitions of of this procedure were conducted for each type of sponge.

\section{Curcumin-release profile}

Cur-release profiles of CA-Cur and CA-CD-Cur (containing $25 \mathrm{mg}$ Cur/six-well plate, ie, $4.17 \mathrm{mg}$ Cur/sponge) were conducted using RP-HPLC. Sponges were placed in six-well plates with $7 \mathrm{~mL}$ PBS (0.01 M, pH 7.4) in each well, and the plates were gently shaken at $37^{\circ} \mathrm{C}$. All supernatants were pipetted out and at predetermined time points replaced with the same volume of prewarmed PBS. After centrifugation at $13,000 \mathrm{rpm}$ for 10 minutes, the supernatant was collected and stored at $-20^{\circ} \mathrm{C}$ until further analysis. Released Cur was quantified at $425 \mathrm{~nm}$ with RP-HPLC (Waters) as described earlier. ${ }^{1}$

\section{Grouping and full-thickness cutaneous rat model}

Based on our preliminary experiment (Figures S2 and S3), an optimal concentration of CA-CD-Cur (25 mg of Cur/six sponges; sponges prepared in six-well plates) was used for this part of experiment. In this part, 48 rats were randomly divided into four groups (12 rats per group: six for the wound-closure study and six for histological and mechanisms study): gauze, CA, CA-Cur, and CA-CD-Cur. Rats were anesthetized, their hair removed, and disinfected. Full-thickness cutaneous wounds $(2 \times 2 \mathrm{~cm})$ were created using a scalpel to the depth of superficial fascia.

\section{Wound-closure rate}

Images of wounds were taken with a Cnon digital camera (IXUS $1100 \mathrm{HS}$ ) on days 3, 7, and 14. Photographs were taken under identical light conditions with ruler stickers placed on the edges of the wounds as measuring scale. The wound area was measured by Photoshop CS 6.0 using the methods of Papazoglous et al: ${ }^{16}$

$$
\text { wound-closure rate }=\left(A_{0}-A_{t}\right) / A_{0} \times 100
$$

where $A_{0}$ is the original wound area and $A_{t}$ the actual area at different time points. Wounds were observed and photographed on days 3,7 , and 14 postwounding.

\section{Histopathological examination}

Granulation tissue was harvested from each group at days 7 and 14 postwounding. Tissues were fixed in $10 \%$ buffered formalin for 24 hours at $4^{\circ} \mathrm{C}$. Fixed samples were then embedded in paraffin and sectioned perpendicularly to the wound surface in $4 \mu \mathrm{M}$ sections. H\&E and Masson's trichrome staining were performed using standard procedures. Histological evaluation was performed by two observers blinded to the treatment using light microscopy. A modified histology scale was used to score the H\&E sections. Degree of granulation tissue, cell infiltration, proportions of inflammatory and proliferative cells, capillary density, and extracellular matrix deposition were the key elements to determine the score.

\section{Sircol collagen assay}

Sircol collagen assays (Biocolor) were conducted to quantify soluble-collagen content at day 14 postwounding supplementary to Masson's trichrome staining: $1 \times 1 \mathrm{~cm}$ strips were harvested from each wound, and samples were then prepared and assayed following the manufacturer's protocol.

\section{Antioxidant-capacity analysis}

On day 7 postoperation, six animals per group were euthanized and granulation-tissue samples collected. For antioxidant analysis, 10\% (w:v) wound-tissue homogenate was prepared in neutral PBS $(50 \mathrm{mM})$ and centrifuged 
(1,000 rpm for 10 minutes at $\left.4^{\circ} \mathrm{C}\right)$. The resultant supernatant was then subjected to superoxide dismutase (SOD), catalase, and lipid-peroxidation assays following the manufacturer' s protocol.

\section{Western blot analysis}

Granulation-tissue samples were collected on day 7 postoperation. Tissue homogenate was prepared in PBS (50 $\mathrm{mM}, \mathrm{pH}$ 7.4) with a homogenizer. The resulting homogenate was centrifuged at $1,000 \mathrm{rpm}$ for 10 minutes. These steps were conducted on ice to avoid protein denaturing and degradation. The resultant supernatant was then subjected to Western blot analysis following a standardized protocol. $^{22}$ Primary antibodies used in this study were anti$\mathrm{I} \kappa \mathrm{B} \alpha$, anti-TGF $\left.\beta_{1}\right)$, anti-pAkt, and anti-PI3K.

\section{Real-time PCR analysis}

Granulation-tissue samples were collected on day 7 postoperation. After samples had been washed with PBS containing DEPC, total RNA was immediately extracted from collected using Trizol reagent. cDNA was generated using a reverse-transcription system (ImProm-II; Promega, Madison, WI, USA). Quantitative real-time PCR was performed using an ABI 7900. Sequences for gene-specific PCR primers were: $\beta$-actin - forward 5'-CACGATGGA GGGGCCGGACTCATC-3', reverse 5'-TAAAGACCTCT ATGCCAACACAGT-3'; NFאB - forward 5'-GGCAGC ACTCCTTATCAACC-'3, reverse 5'-GAGGTGTCGTCC CATCGTAG-'3; and TGF $\beta_{1}$ - forward 5'-GTCAACT GTGGAGCAACACG-3', reverse 5'-ACTGAAGCGAAA GCCCTGTA-3.

\section{Statistical analysis}

Data are expressed as means \pm SD. Data analysis was carried out using GraphPad Prism. Comparisons of experimental data among different groups were conducted using one-way ANOVA with the Student-Newman-Keuls test applied post hoc for paired comparisons of means or Mann-Whitney $U$ test. $P<0.05$ (two-tailed) was considered statistically significant.

\section{Results}

\section{Physicochemical characterization of CA,} CA-Cur, and CA-CD-Cur sponges

As shown in Figure 1, CA (Figure 1A), CA-Cur (Figure 1B), and CA-CD-Cur (Figure 1C) sponges were successfully prepared. The sponges were light and flexible.
Figure 1D-F shows the porous structure of the sponges. In contrast to the tabular network of CA (Figure 1D), CA-Cur (Figure 1E) exhibited a much more fibrillar structure, which might be attributed to the lyophobic nature of Cur. With improvement in hydrophilicity of CD-Cur, CA-CDCur (Figure 1F) exhibited a similar structure to CA.

All three kinds of sponge showed superior wateruptake capacity (Figure 1G) due to the porous structure. Specifically, CA-Cur held much less water $(2,133.50 \%$ $\pm 355.69 \%)$ than CA $(4,023 \% \pm 435.26 \% ; P<0.01$ on oneway ANOVA) alone. This might be explained by the lyophobic nature of Cur. With improved hydrophilia of CD-Cur, CA-CD-Cur absorbed more water $(3,274.5 \%$ $\pm 405.35 \%, P<0.01$ on one-way ANOVA) than CA-Cur.

Figure $1 \mathrm{H}$ depicts the degradation profile of different sponges. After 14 days, all sponges were partially degraded, from 50\% (CA-CD-Cur $48.76 \% \pm 1.57 \%$ ) to $25 \%$ (Cur $27.25 \% \pm 1.60 \%$ ). Figure 1 I shows the drugrelease profile of $\mathrm{CA}-\mathrm{Cur}$ and $\mathrm{CA}-\mathrm{CD}-\mathrm{Cur}$. Cur was released in a sustained manner from CA-Cur and CA-CD-Cur. Within 14 days, more Cur $(60.61 \% \pm 5.96 \%)$ was released from CA-CD-Cur than CA-Cur (39.34\% $\pm 6.48 \% ; P<0.05$, independent-sample $t$-test).

\section{CA-CD-Cur-treated wounds got higher granulation-tissue scores in the early stage of wound healing}

Figure 2 shows representative H\&E sections for granulation-tissue scoring. As we can see, there were limited inflammatory cells presented in gauze-treated wounds (Figure 2A). CA-treated wounds (Figure 2B) showed immature granulation tissue with numerous cells, though these were mainly inflammatory. In contrast, CA-Curtreated wounds (Figure 2C) exhibited loose reticular tissue infiltrated with inflammatory and proliferative cells. Mild neovascularization was also observed. Last but not least, CA-CD-Cur-treated wounds (Figure 2D) showed massive vascularized granulation tissue with numerous cells, among which most were fibroblasts.

Based on Spenny's modified histology scale, CA-CDCur-treated wounds got the highest score $(12.33 \pm 1.37$, Figure 2E), characterized by vascular granulation tissue filled with fibroblasts and quite a few collagen depositions compared with the CA-Cur $(10.33 \pm 1.37, P<0.05$, MannWhitney $U$ test $), C A(9 \pm 1.79, P<0.05$, Mann-Whitney $U$ test), and gauze groups $(6.83 \pm 1.47, P<0.01$, MannWhitney $U$ test). 

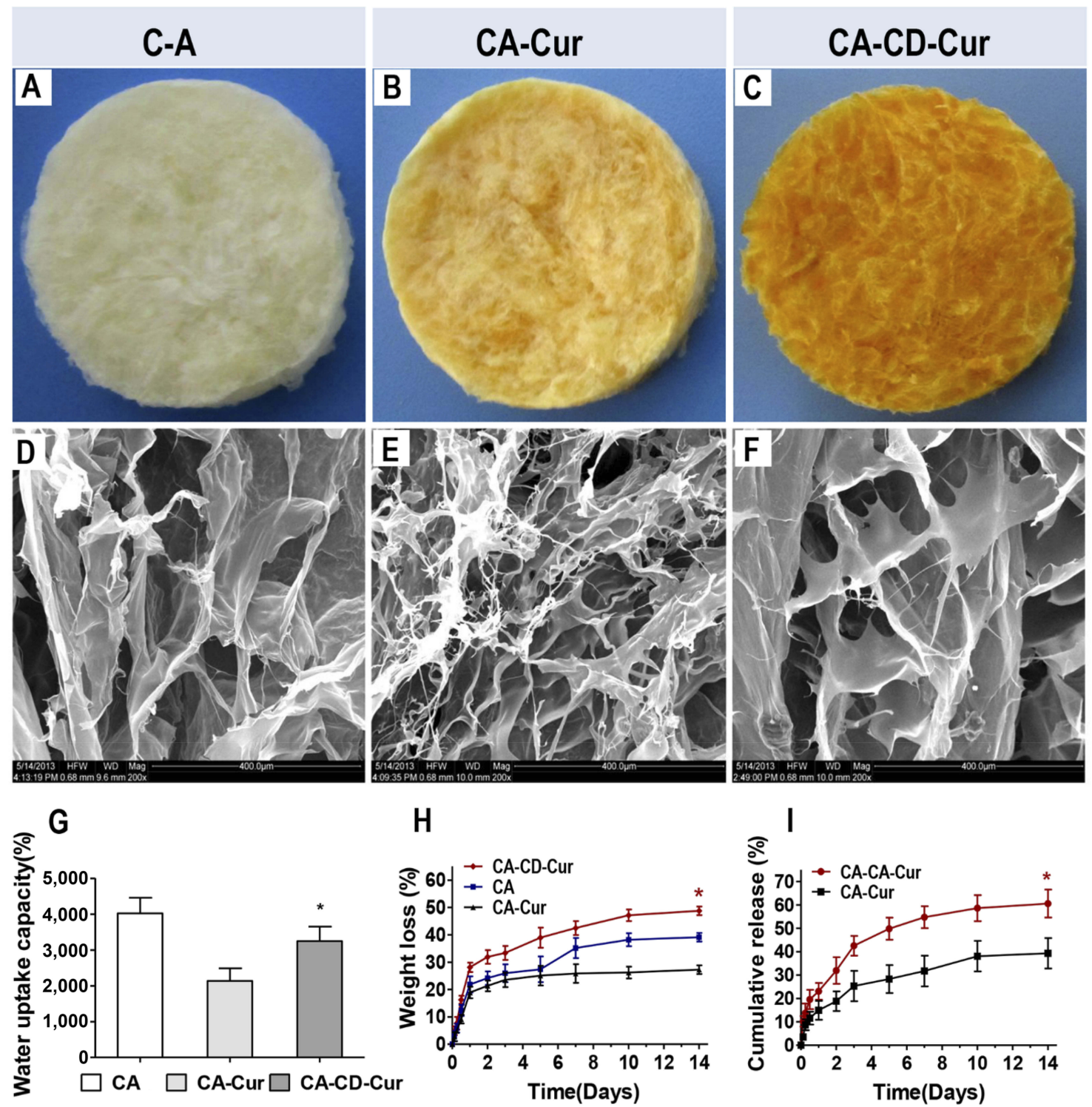

Figure I Physicochemical characterization of CA, CA-Cur, and CA-CD-Cur.

Notes: (A-F) Macro- and microappearance of CA, CA-Cur, and CA-CD-Cur; $(\mathbf{G}, \mathbf{H})$ in vitro water-uptake ability and degradation profile of different sponges; (I) curcumin-release profiles of CA-Cur and CA-CD-Cur. Data shown as means $\pm S D(n=3) ; * P<0.05$ (one-way ANOVA).

\section{CA-CD-Cur accelerated cutaneous wound healing in rats}

The wound healing capacity of gauze, CA, CA-Cur, and CA-CD-Cur is shown in Figure 3. No distinct irritative or infectious signs were observed among any of the four groups. CA-CD-Cur-treated wounds exhibited the fastest healing $(60.24 \% \pm 5.81 \%$ on day $7,94.39 \% \pm 5.40 \%$ on day 14) compared to CA $(53.49 \% \pm 6.74 \%$ on day $7,80.40 \%$ $\pm 9.01 \%$ on day $14 ; P<0.05$, one-way ANOVA), and gauze $(40.41 \% \pm 4.64 \%$ on day $7,64.55 \% \pm 7.84 \%$ on day $14 ; P<0.05)$. However, no significant difference was observed between CA-CD-Cur and CA-Cur (60.72\% $\pm 4.87 \%$ on day $7,92.42 \% \pm 6.24 \%$ on day $14 ; P<0.05$ ). No significant differences were observed among groups on day 3 .

\section{CA-CD-Cur-treated wounds showed advanced reepithelialization}

Figure 4 shows the histopathological profile of wounds treated with gauze, CA, CA-Cur, or CA-CD-Cur on day 14 postoperation. As shown in Figure 4D, wounds treated with CA-CD-Cur showed advanced reepithelialization, well-organized dermis (cells and collagen fibers were compact and well aligned), and more extracellular matrix 


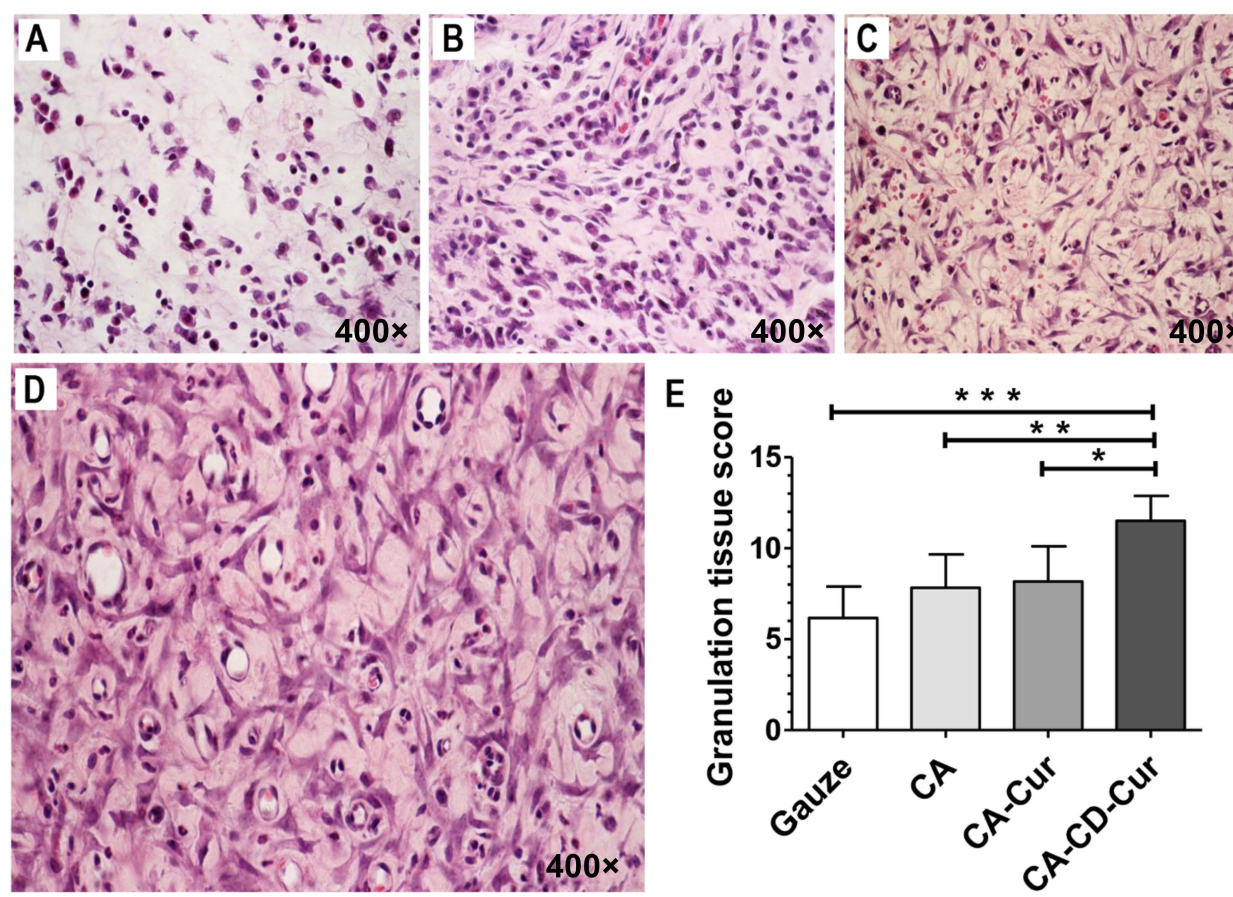

Figure 2 CA-CD-Cur facilitated granulation-tissue maturation by accelerating the transition process from the inflammation phase to the proliferation phase. Notes: Representative H\&E sections of granulation tissue treated with gauze (A), CA (B), CA-Cur (C), and CA-CD-Cur (D). Tissue samples were harvested from the center of each wound at day 7 postoperation. (E) Granulation tissue-score comparison among different groups. Data shown as means $\pm S D$ ( $n=6$ ). $* P<0.05$, $* * P<0.0$ I, $* * * P<0.00$ I, one-way ANOVA.

deposition compared with the CD-Cur, CA, and gauze groups. While most sections in the gauze- and CA-treated groups showed inferior epithelialization, both CA-Curand CA-CD-Cur-treated sections were characterized by epithelia with multiple layers of cells. Despite the fact that epithelial thickness was greater in CD-Cur-treated sections than those treated with CA-CD-Cur, CA-CDCur-treated sections showed well-structured epidermises with three to five layers of epithelial cells, which was similar to epithelia found in physiological conditions.

\section{CA-CD-Cur-treated wounds showed more collagen deposition and better collagen-fiber alignment}

As shown in Figure 5, CA-CD-Cur-treated wounds showed more densely packed collagen bundles than other groups. Additionally, Sircol collagen assay (Figure 5E) show that CA-CD-Cur-treated wounds contained the highest levels of collagen $(44.3 \pm 2.6 \mu \mathrm{g} / \mathrm{mg})$ compared to CA-Cur $(36.4 \pm 6.2$ $\mu \mathrm{g} / \mathrm{mg}, P<0.01$, one-way ANOVA), CA $(33.4 \pm 3.7 \mu \mathrm{g} / \mathrm{mg}$, $P<0.05$, one-way ANOVA), and gauze $(32.2 \pm 2.3 \mu \mathrm{g} / \mathrm{mg}$, $P<0.05$, one-way ANOVA) on day 14 .

\section{CA-CD-Cur significantly improved wound} microenvironment by reducing oxidative stress, downregulating NFKB-signaling pathway, and improving TGF $\beta_{\text {I }}$ expression Local SOD, catalase, and malondialdehyde (product of lipid peroxidation) levels were assayed to evaluate oxidative stress at wound sites. As shown in Figure 6A-C, SOD and malondialdehyde levels were lower in Cur-treated (CA-Cur and CA-CD-Cur) groups than Cur-free (CA and gauze) groups. Moreover, minimum SOD levels were observed in CA-CD-Cur-treated wounds. On the other hand, catalase was highest in the CA-CD-Cur-treated group.

As shown in Figure 6D, on day 7 postoperation, reduced expression of PI3K and pAkt can be seen in the Western blot bands of CA-CD-Cur, while I $\mathrm{B} \alpha$ (inhibitory protein of the PI3K-Akt-NFkB signaling pathway) expressed more than other groups. Realtime PCR results (Figure 6E) indicated significantly reduced expression of $\mathrm{NF} \kappa \mathrm{B}$ mRNA. Increased levels of TGF $\beta_{1}$ were also observed at both protein (Figure 6F) and mRNA (Figure 6G) levels. 
A
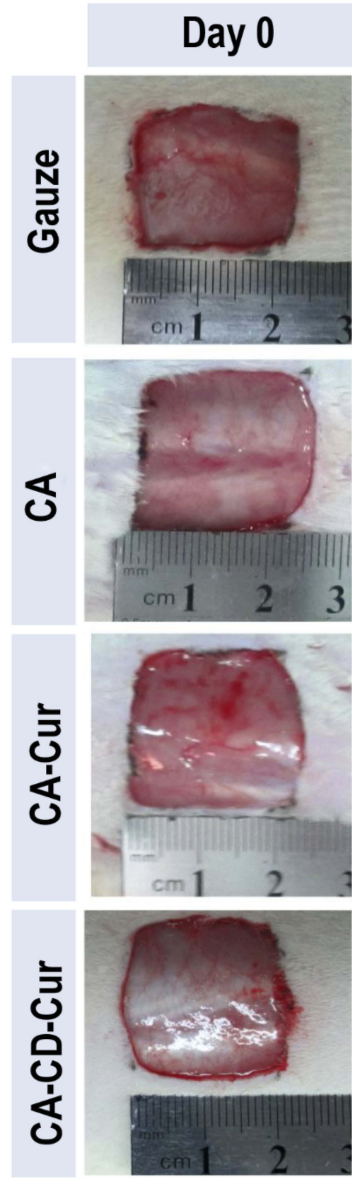

B
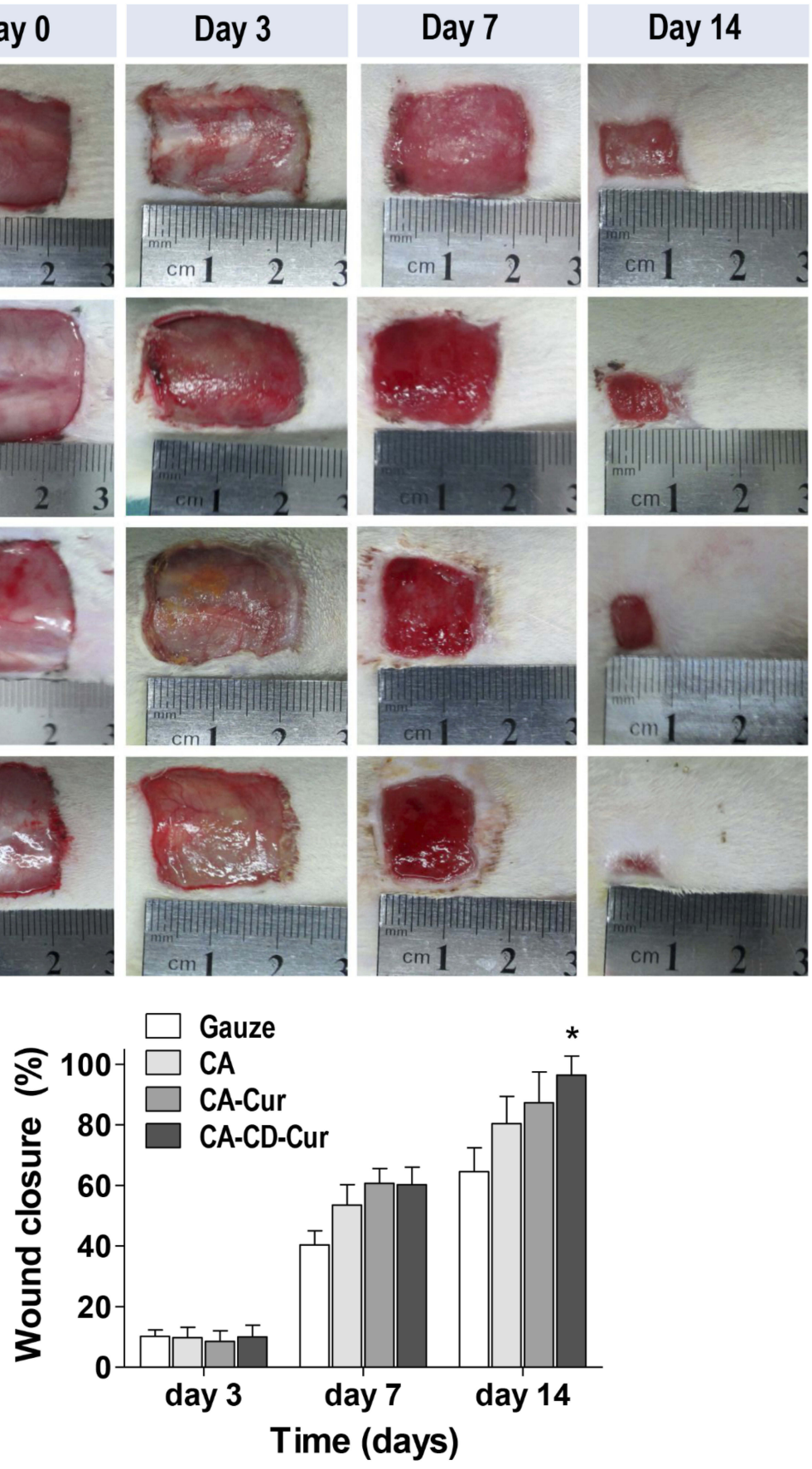

Figure 3 CA-CD-Cur-treated wounds showed accelerated wound closure compared to gauze-, CA-, and CA-Cur-treated wounds.

Notes: (A) Representative images of wounds with different treatments at day 0 , day 3, day 7, and day 14 postoperation; (B) wound-closure comparison among groups at different time points. Data shown as means $\pm S D(n=6)$. $* P<0.05$ (one-way ANOVA).

\section{Discussion}

In the present study, we developed a novel Cur-loaded composite dressing: CA-CD-Cur. CA-CD-Cur was successfully prepared and characterized to be a suitable wound-dressing material with controlled release, suitable degradation rate, and water-uptake ability. In vivo animal experiments showed that
CA-CD-Cur-dressed wounds healed faster than other groups in full-thickness-excision rat models. Superior outcomes were achieved at both histological and molecular levels.

Cur has long been used to treat various disease, including cutaneous wounds. However, Cur's hydrophobic nature significantly limits its application. In this study, we adopted CD- 

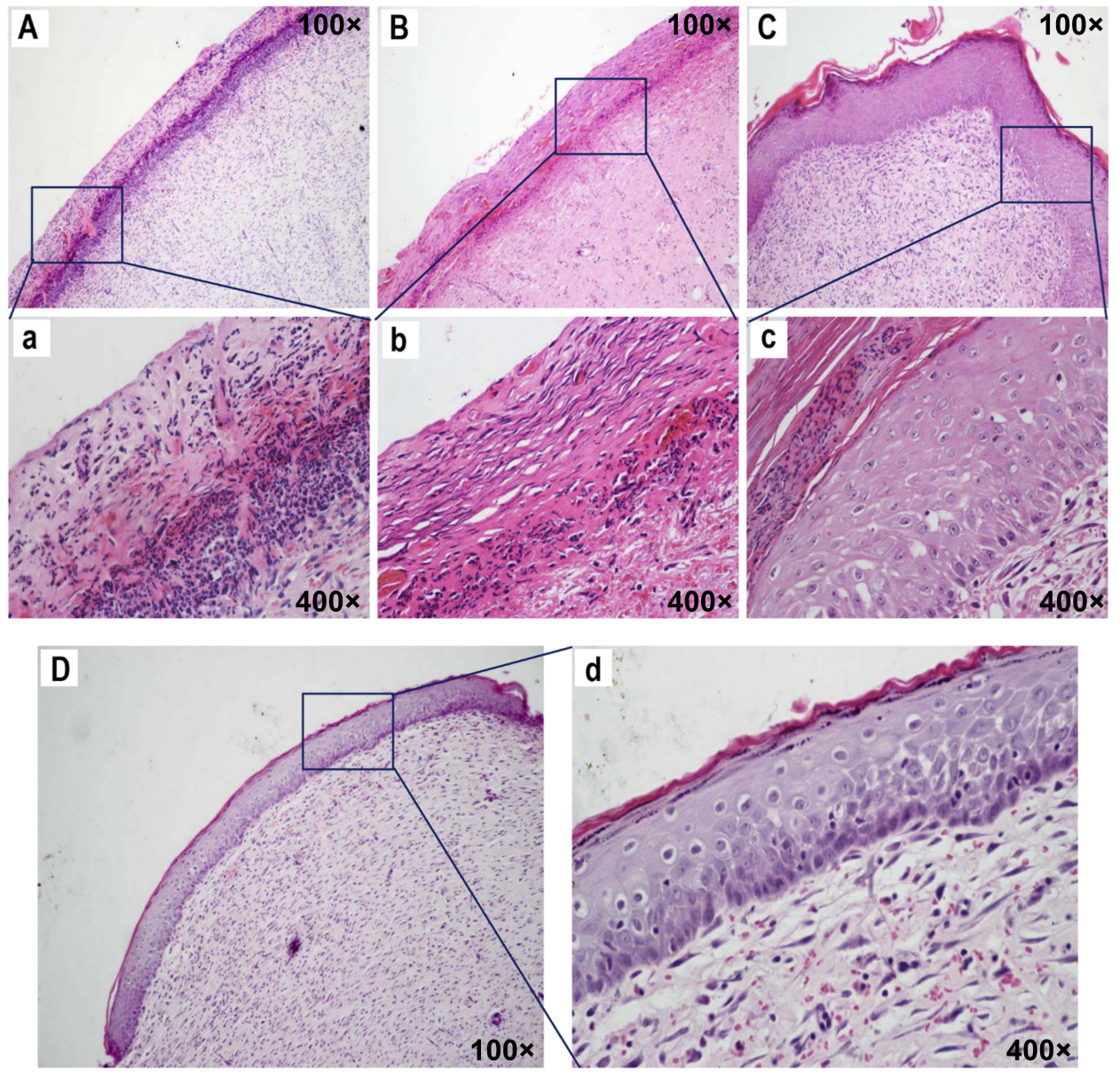

Figure 4 CA-CD-Cur-treated wounds showed optimized epithelialization.

Notes: Representative H\&E images of granulation tissue treated with gauze (A), CA (B), CA-Cur (C), and CA-CD-Cur (D). Tissue samples were harvested from the center of each wound at day 14 postoperation.

Cur as the curcumin-modified form, which is known for its improved water solubility, stability, and pharmacological effects compared to curcumin. ${ }^{10,17}$ As shown in Figure 7, via self-assembly, hydrophobic Cur molecules (Figure 7C) were included in the ring-structured CD molecule (Figure 7D), which has a hydrophilic outer surface and hydrophobic inner surface. ${ }^{18-20}$ Meanwhile, positively charged chitosan molecules (Figure 7A) were mixed with negatively charged alginate molecules (Figure 7B) to form CA. Finally, CD-Cur was then added to the CA solution to form the porous CA-CD-Cur (Figure 7, E and F) via freeze-drying.

In contrast to CA-Cur, CA-CD-Cur exhibited a tabular inner structure (compared to the fibrillar structure of CA-Cur), superior water-uptake capacity, and faster Cur-release profile. These results can be explained by the improved hydrophilic property because of the inclusion of Cur by cyclodextrin. ${ }^{9,21-23}$ Cur-containing sponge (CA-Cur and $\mathrm{CA}-\mathrm{CD}-\mathrm{Cur}$--treated wounds resulted in faster wound healing and advanced histological results compared with CA- and gauze-treated wounds. This might largely be due to the wound-healing capacity of Cur, which has been reported before. 1,13,24 Furthermore, CA-CD-Cur-treated wounds were even better than CA-Cur, which we may attribute to the superior water solubility and cell-uptake capacity of CD-Cur. ${ }^{10,17,23,25}$ However, despite its improved bioactivity, CA-CD-Cur was slightly more fragile than CACur in terms of physical strength, especially when completely dry (when coming out of the chamber of the freezedrier). Additionally, CA-CD-Cur degraded faster than CACur, which may also be attributable to the enhanced water solubility of CD-Cur. ${ }^{9,10,12}$

Cutaneous wound healing consists of three interrelated stages: inflammatory, proliferation, and remodeling. In that context, we discuss how CA-CD-Cur's wound-healing capacity is incorporated into the three phases, with special focus on the inflammation phase, during which most of the 

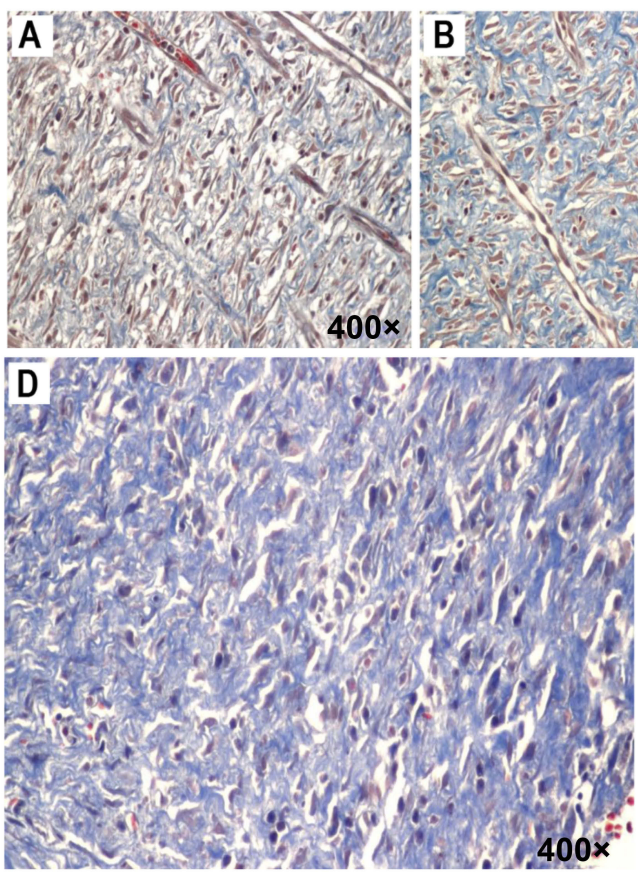
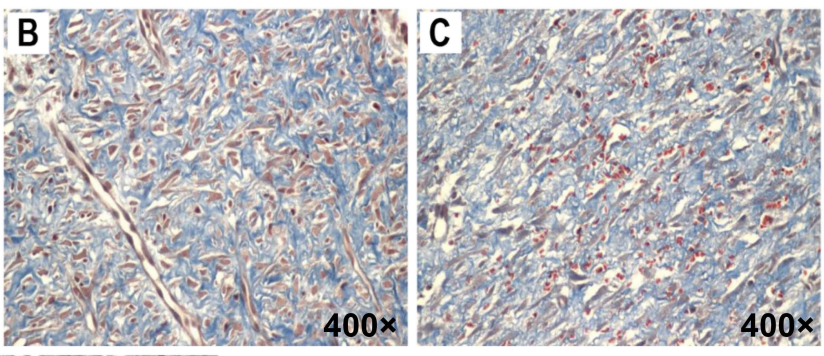

E

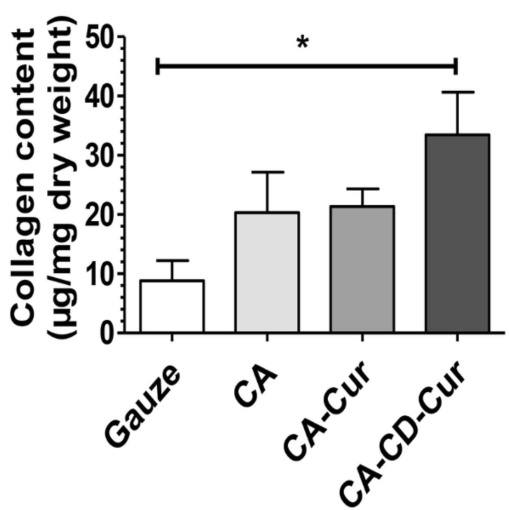

Figure 5 CA-CD-Cur-treated wounds led to more abundant collagen deposition with superior alignment.

Notes: Representative Masson's trichrome-stained sections of granulation tissue treated with gauze (A), CA (B), CA-Cur (C), or CA-CD-Cur (D) at day I4 postoperation. (E) Sircol collagen-assay results. Data shown as means \pm SD $(n=3)$. *P<0.05 (one-way ANOVA).

biological change occurs. During the inflammatory phase, various radicals are generated due to the injury. ${ }^{1-3}$ Radicals are often associated with oxidative stress, which leads to lipid peroxidation and impaired wound healing. ${ }^{1,3}$ SOD and catalase are enzymes that help in scavenging excessive radicals, hence levels of SOD and catalase would somehow reflect the level of oxidative stress. Decreased oxidative stress optimizes the inflammatory response and thus inhibits the transcription and downregulation of $\mathrm{NF \kappa B}$, thus leading to the low gene expression of $S O D .^{32}$ In the present study, SOD and lipid peroxidation were dramatically lower in Cur-containing sponge-treated groups than the CA and gauze groups, which might indicate a microenvironment with less oxidative stress. The result proves that Cur did have the ability to scavenge radicals, as previously reported both in vitro and in in vivo. ${ }^{1,3,26}$ Moreover, CA-CD-Cur-treated wounds exhibited even lower levels of SOD and lipid peroxidation than CA-Cur-treated ones, which may be attributable to the higher water solubility and cell-uptake capacity of the CD-Cur inclusion complex. ${ }^{10,17}$ On the other hand, catalase levels were decreased in the Cur-treated group. This was because scavenged superoxide radicals were converted into hydrogen peroxide, which then stimulated the expression of catalase. Our results match well with former research. $^{2,24}$

$\mathrm{NF \kappa B}$ is a transcription factor that helps to govern the expression of genes involved in immuno- and inflammatory response. NFkB can be activated by various of stimuli, eg, oxidative stress. In brief, radicals initially phosphorylate PI3K/ Akt. NFкB is then activated by phosphorylation and subsequent degradation of an inhibitory protein: IкB (a subunit of

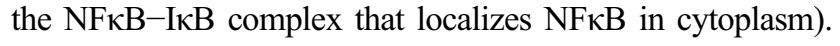
Consequently, $\mathrm{NF \kappa B}$ enters the the nucleus resulting in the expression of target genes. ${ }^{27,28}$ Consistently with former studies involving Cur and the NFkB signaling pathway, ${ }^{1,29}$ lower expression of PI3K and pAkt was seen in CA-CD-Cur-treated groups in our Western blot results, whereas IKB was upregulated. This result was further confirmed by RT-PCR, which showed downregulation of $\mathrm{NF \kappa B}$. Our results indicated that CA-CD-Cur might attenuate inflammation through the NFkBsignaling pathway, partly due to sufficient radical-scavenging capacity.

Expressed by monocytes and macrophages, TGF $\beta_{1}$ has long been known to facilitate wound healing via many cellular processes including embryogenesis, inflammation, immunoresponses, and tissue repair. Diverse effects of TGF $\beta_{1}$ were exerted on different cell types, including 

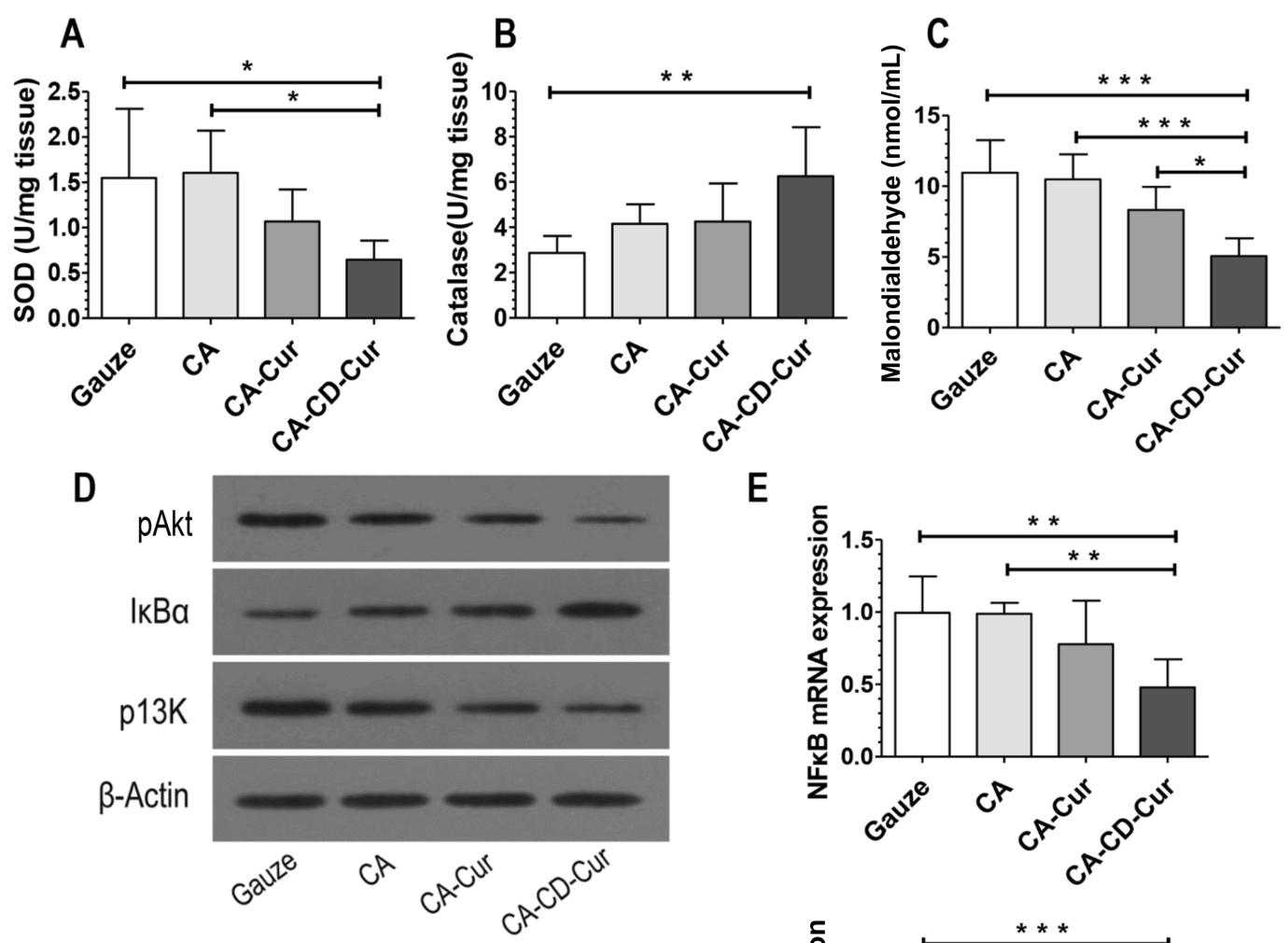

$\mathrm{E}$

$\mathbf{F}$
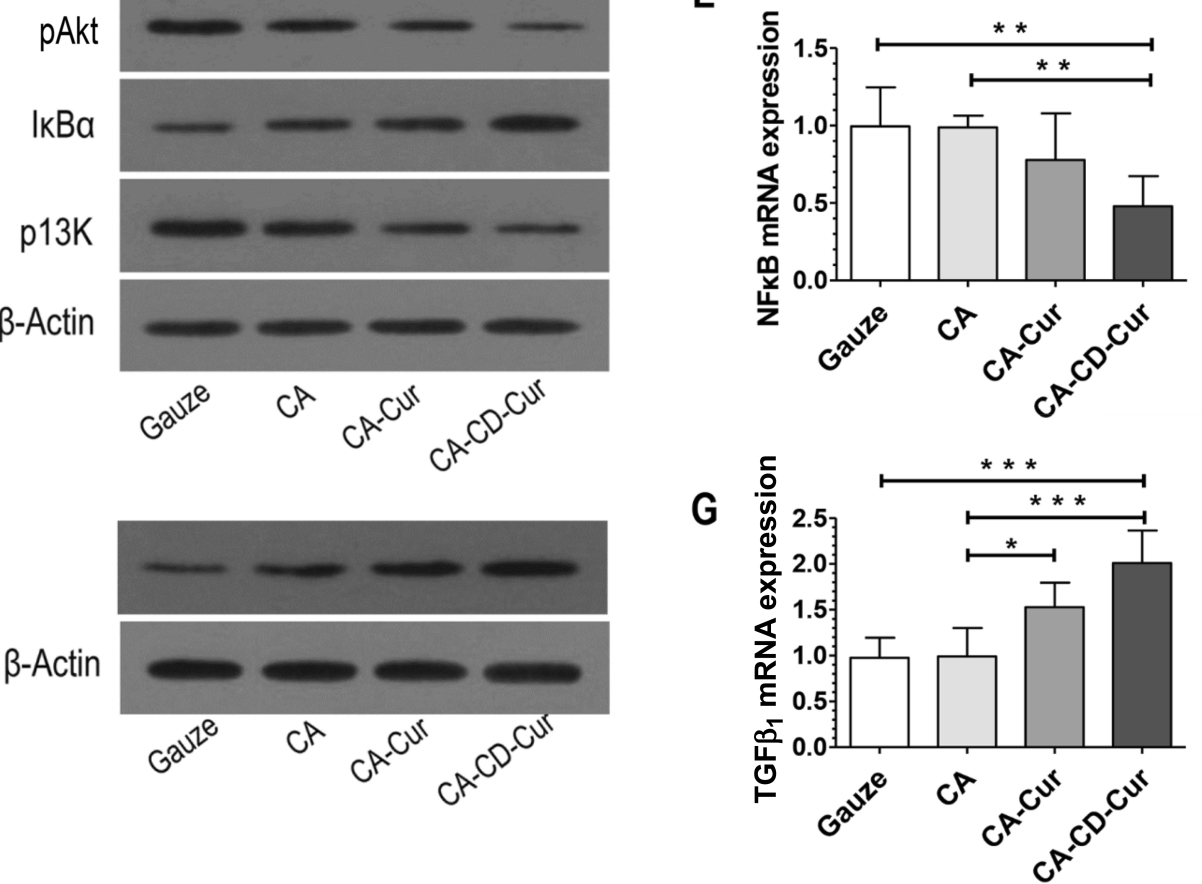

Figure 6 CA-CD-Cur improved the wound microenvironment by reducing oxidative stress (A-C), lowing inflammatory factors (D and , E) and consequently elevating $\mathrm{TGF}_{\text {I }}$ ( $\mathbf{F}$ and, $\mathbf{G}$ ).

Notes: Data shown as means $\pm S D(n=6) . * P<0.05$, **P<0.01, *** $P<0.001$ (one-way ANOVA).

keratinocytes, fibroblasts, inflammatory cells, and endothelial cells. ${ }^{30,31} \mathrm{TGF}_{1}$ can also stimulate fibroblasts from the tissue around the wound to proliferate, express appropriate integrin receptors, migrate into the wound site, and thus facilitate wound healing. ${ }^{31,32}$ Cur has been identified as a wound-healing agent in cutaneous wounds by facilitating TGF $\beta_{1}$ generation in both normal- and impairedhealing wounds. ${ }^{33-35}$ In the present study, increased levels of TGF $\beta_{1}$ protein as well as mRNA were observed in Cur-treated wounds compared to non-Cur groups. In particular, CA-CD-Cur-treated wounds showed even higher $\mathrm{TGF} \beta_{1}$ levels. This increased level was presumably due to the enhanced water solubility of $\mathrm{CD}$-Cur, as well as increased cell-uptake capacity. ${ }^{10,17}$
Following the inflammatory phase is the proliferation phase, with granulation-tissue formation. Our histological results showed that the CA-CD-Cur-treated group got higher wound-maturity scores at day 7 postoperation, with more fibroblasts and newly formed capillaries. More nutrients were thus delivered to the wound site, along with more extracellular matrix deposition. ${ }^{31,32}$ As shown in Figure 7A, CA-CD-Curtreated wounds showed the highest granulation-tissue scores, probably due to the less oxidative and inflammatory environment.

Finally, we enter the remodeling phase, where significant wound contraction and extracellular matrix reorganization take place. Minimum wound area and well-organized granulation tissue were achieved in CA-CD-Cur-treated wounds 


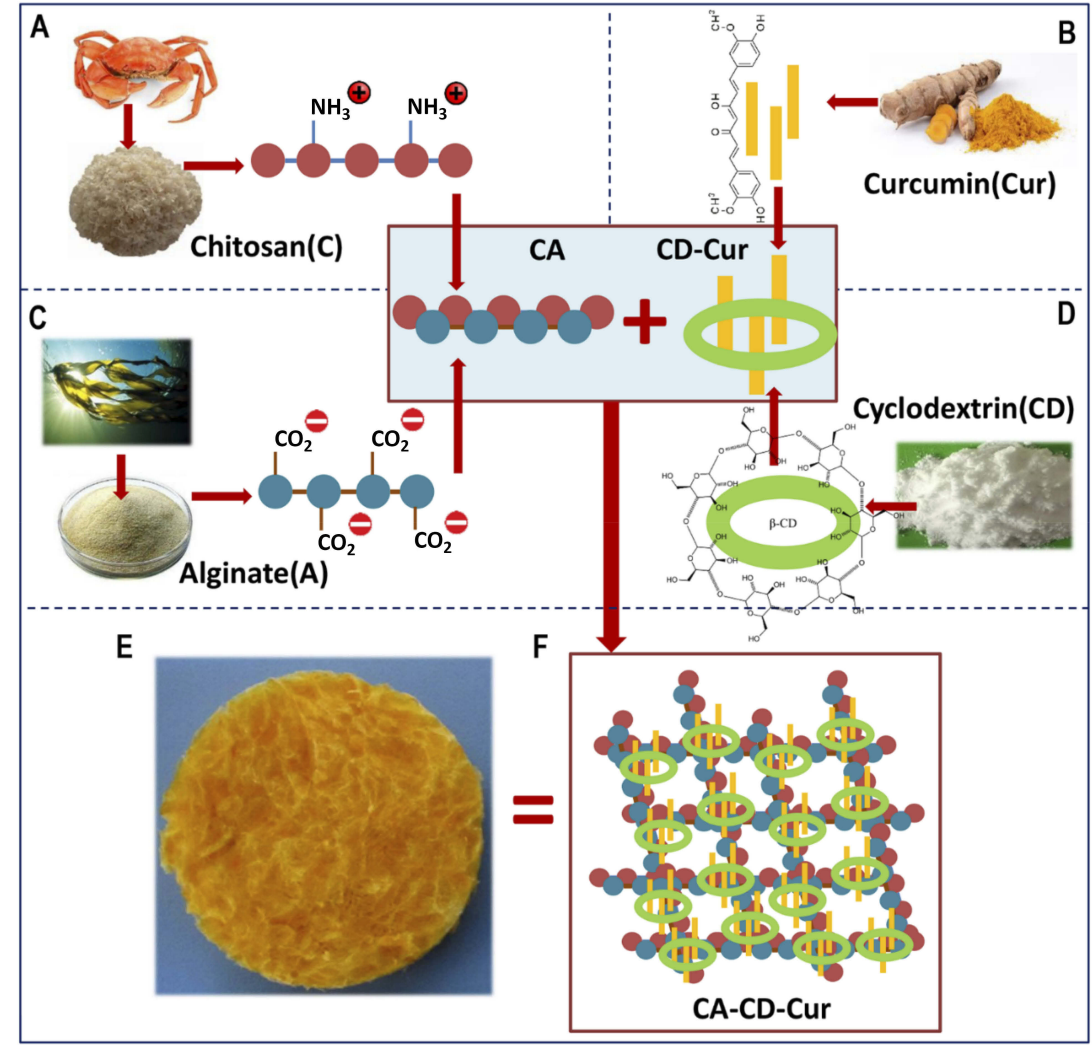

Figure 7 Illustration of the process of making CA-CD-Cur.

Notes: (A) Positively charged chitosan; (B) negatively charged alginate; (C) hydrophobic Cur; (D) ring-shaped CD; (E, F) CA-CD-Cur with porous structure.

on day 7 postoperation. Previous research has suggested that Cur is able to modulate collagen deposition. Consistently with this, our Masson staining and Sircol collagen assay firmly supported that better-structured collagen fibers had deposited in granulation tissue covered by CA-CD-Cur in comparison with other groups.

\section{Conclusion}

In the present work, CA-CD-Cur was successfully prepared and characterized to be a suitable wound-dressing material with suitable drug release, degradation rate, and water uptake. Animal studies showed that CA-CD-Cur is able to facilitate cutaneous wound healing. Possible mechanisms of action involved in this accelerated wound-healing process may be attributed to the advanced antioxidative, anti-inflammatory, and $\mathrm{TGF} \beta_{1}$-inducing capacity of the CA-CD-Cur dressing.

\section{Acknowledgment}

This work was financially supported by the National Natural Science Foundation of China (81572163), the Fundamental Research Fund for the Central Universities, and the Zhongnan Hospital of Wuhan University Science,
Technology, and Innovation Seed Fund (cxpy20160014). YZ and CYD share joint first authorship, as they contributed equally to this work.

\section{Disclosure}

The authors report no conflicts of interest in this work.

\section{References}

1. Mohanty C, Das M, Sahoo SK. Sustained wound healing activity of curcumin loaded oleic acid based polymeric bandage in a rat model. Mol Pharm. 2012;9(10):2801-2811. doi:10.1021/mp300075u

2. Gopinath D, Ahmed MR, Gomathi K, Chitra K, Sehgal PK, Jayakumar R. Dermal wound healing processes with curcumin incorporated collagen films. Biomaterials. 2004;25(10):1911-1917. doi:10.1016/ s0142-9612(03)00625-2

3. Thangapazham RL, Sharad S, Maheshwari RK. Skin regenerative potentials of curcumin. Biofactors. 2013;39(1):141-149. doi:10.1002/biof.1078

4. Kulac M, Aktas C, Tulubas F, et al. The effects of topical treatment with curcumin on burn wound healing in rats. $J$ Mol Histol. 2013;44 (1):83-90. doi:10.1007/s10735-012-9452-9

5. Wang WY, Cao YX, Zhou X, Wei B. Delivery of folic acid-modified liposomal curcumin for targeted cervical carcinoma therapy. Drug Des Devel Ther. 2019;13:2205-2213. doi:10.2147/DDDT.S205787

6. Jagetia GC, Rajanikant GK. Acceleration of wound repair by curcumin in the excision wound of mice exposed to different doses of fractionated gamma radiation. Int Wound J. 2012;9(1):76-92. doi:10.1111/ j.1742-481X.2011.00848.x 
7. Lopez-Jornet P, Camacho-Alonso F, Jimenez-Torres MJ, OrdunaDomingo A, Gomez-Garcia F. Topical curcumin for the healing of carbon dioxide laser skin wounds in mice. Photomed Laser Surg. 2011;29(12):809-814. doi:10.1089/pho.2011.3004

8. Boztas AO, Karakuzu O, Galante G, et al. Synergistic interaction of paclitaxel and curcumin with cyclodextrin polymer complexation in human cancer cells. Mol Pharm. 2013;10(7):2676-2683. doi:10.10 21/mp400101k

9. Kaur N, Garg T, Goyal AK, Rath G. Formulation, optimization and evaluation of curcumin-beta-cyclodextrin-loaded sponge for effective drug delivery in thermal burns chemotherapy. Drug Deliv. 2016;23 (7):2245-2254. doi:10.3109/10717544.2014.963900

10. Yallapu MM, Jaggi M, Chauhan SC. beta-cyclodextrin-curcumin self-assembly enhances curcumin delivery in prostate cancer cells. Colloids Surf B Biointerfaces. 2010;79(1):113-125. doi:10.1016/j. colsurfb.2010.03.039

11. Yadav VR, Prasad S, Kannappan R, et al. Cyclodextrin-complexed curcumin exhibits anti-inflammatory and antiproliferative activities superior to those of curcumin through higher cellular uptake. Biochem Pharmacol. 2010;80(7):1021-1032. doi:10.1016/j.bcp.2010.06.022

12. Rachmawati H, Edityaningrum CA, Mauludin R. Molecular inclusion complex of curcumin-beta-cyclodextrin nanoparticle to enhance curcumin skin permeability from hydrophilic matrix gel. AAPS PharmSciTech. 2013;14(4):1303-1312. doi:10.1208/s12249-013-0023-5

13. Dai M, Zheng X, Xu X, et al. Chitosan-alginate sponge: preparation and application in curcumin delivery for dermal wound healing in rat. J Biomed Biotechnol. 2009;2009:595126. doi:10.1155/2009/595126

14. Wu T, Huang J, Jiang Y, et al. Formation of hydrogels based on chitosan/alginate for the delivery of lysozyme and their antibacterial activity. Food Chem. 2018;240:361-369. doi:10.1016/j. foodchem.2017.07.052

15. Shen H, Li F, Wang D, et al. Chitosan-alginate BSA-gel-capsules for local chemotherapy against drug-resistant breast cancer. Drug Des Devel Ther. 2018;12:921-934. doi:10.2147/DDDT.S158001

16. Papazoglou ES, Zubkov L, Mao X, Neidrauer M, Rannou N, Weingarten MS. Image analysis of chronic wounds for determining the surface area. Wound Repair Regen. 2010;18(4):349-358. doi:10.1111/j.1524-475X.2010.00594.x

17. Tomren MA, Masson M, Loftsson T, Tonnesen HH. Studies on curcumin and curcuminoids XXXI. Symmetric and asymmetric curcuminoids: stability, activity and complexation with cyclodextrin. Int J Pharm. 2007;338(1-2):27-34. doi:10.1016/j.ijpharm.2007.01.013

18. Sudakaran SV, Venugopal JR, Vijayakumar GP, Abisegapriyan S, Grace AN, Ramakrishna S. Sequel of $\mathrm{MgO}$ nanoparticles in PLACL nanofibers for anti-cancer therapy in synergy with curcumin/beta-cyclodextrin. Mater Sci Eng C Mater Biol Appl. 2017;71:620-628. doi:10.1016/j.msec.2016.10.050

19. Zhang L, Man S, Qiu H, et al. Curcumin-cyclodextrin complexes enhanced the anti-cancer effects of curcumin. Environ Toxicol Pharmacol. 2016;48:31-38. doi:10.1016/j.etap.2016.09.021

20. Aadinath W, Bhushani A, Anandharamakrishnan C. Synergistic radical scavenging potency of curcumin-in-beta-cyclodextrin-in-nanomagnetoliposomes. Mater Sci Eng C Mater Biol Appl. 2016;64:293-302. doi:10.1016/j.msec.2016.03.095

Drug Design, Development and Therapy

\section{Publish your work in this journal}

Drug Design, Development and Therapy is an international, peerreviewed open-access journal that spans the spectrum of drug design and development through to clinical applications. Clinical outcomes, patient safety, and programs for the development and effective, safe, and sustained use of medicines are a feature of the journal, which has also
21. Hegge AB, Andersen T, Melvik JE, Bruzell E, Kristensen S, Tonnesen HH. Formulation and bacterial phototoxicity of curcumin loaded alginate foams for wound treatment applications: studies on curcumin and curcuminoides XLII. J Pharm Sci. 2011;100(1):174185. doi: $10.1002 /$ jps. 22263

22. Yoon SJ, Hyun H, Lee DW, Yang DH. Visible light-cured glycol chitosan hydrogel containing a beta-cyclodextrin-curcumin inclusion complex improves wound healing in vivo. Molecules. 2017;22 (9):1513. doi: $10.3390 /$ molecules22091513

23. Ndong Ntoutoume GM, Granet R, Mbakidi JP, et al. Development of curcumin-cyclodextrin/cellulose nanocrystals complexes: new anticancer drug delivery systems. Bioorg Med Chem Lett. 2016;26 (3):941-945. doi:10.1016/j.bmcl.2015.12.060

24. Gong C, Wu Q, Wang Y, et al. A biodegradable hydrogel system containing curcumin encapsulated in micelles for cutaneous wound healing. Biomaterials. 2013;34(27):6377-6387. doi:10.1016/j. biomaterials.2013.05.005

25. Maria DN, Mishra SR, Wang L, et al. Water-soluble complex of curcumin with cyclodextrins: enhanced physical properties for ocular drug delivery. Curr Drug Deliv. 2017;14(6):875-886. doi:10.2174/ 1567201813666160808111209

26. Phan TT, See P, Lee ST, Chan SY. Protective effects of curcumin against oxidative damage on skin cells in vitro: its implication for wound healing. $J$ Trauma. 2001;51(5):927-931. doi:10.1097/ 00005373-200111000-00017

27. Bowie A, O'Neill LA. Oxidative stress and nuclear factor-kappaB activation: a reassessment of the evidence in the light of recent discoveries. Biochem Pharmacol. 2000;59(1):13-23. doi:10.1016/ s0006-2952(99)00296-8

28. Frey RS, Malik AB. Oxidant signaling in lung cells. Am J Physiol Lung Cell Mol Physiol. 2004;286(1):L1-L3. doi:10.1152/ajplung. 00337.2003

29. Mohanty C, Sahoo SK. The in vitro stability and in vivo pharmacokinetics of curcumin prepared as an aqueous nanoparticulate formulation. Biomaterials. 2010;31(25):6597-6611. doi:10.1016/j. biomaterials.2010.04.062

30. Hong HJ, Jin SE, Park JS, Ahn WS, Kim CK. Accelerated wound healing by smad3 antisense oligonucleotides-impregnated chitosan/ alginate polyelectrolyte complex. Biomaterials. 2008;29(36):48314837. doi:10.1016/j.biomaterials.2008.08.023

31. Gurtner GC, Werner S, Barrandon Y, Longaker MT. Wound repair and regeneration. Nature. 2008;453(7193):314-321. doi:10.1038/ nature 07039

32. Singer AJ, Clark RA. Cutaneous wound healing. $N$ Engl $J$ Med. 1999;341(10):738-746. doi:10.1056/NEJM199909023411006

33. Misra HP, Fridovich I. The role of superoxide anion in the autoxidation of epinephrine and a simple assay for superoxide dismutase. $J$ Biol Chem. 1972;247(10):3170-3175.

34. Aebi H, Scherz B, Ben-Yoseph Y, Wyss SR. Dissociation of erythrocyte catalase into subunits and their re-association. Experientia. 1975;31(4):397-399. doi:10.1007/bf02026338

35. Ohkawa H, Ohishi N, Yagi K. Assay for lipid peroxides in animal tissues by thiobarbituric acid reaction. Anal Biochem. 1979;95 (2):351-358. doi:10.1016/0003-2697(79)90738-3

been accepted for indexing on PubMed Central. The manuscrip management system is completely online and includes a very quick and fair peer-review system, which is all easy to use. Visit http://www. dovepress.com/testimonials.php to read real quotes from published authors. 